\title{
Parameterizing Pair Approximations for Takeover Dynamics
}

\author{
Joshua L. Payne \\ Dept. of Computer Science \\ The University of Vermont \\ Burlington, Vermont 05405 \\ 802-656-9116 \\ Joshua.Payne@uvm.edu
}

\author{
Margaret J. Eppstein \\ Dept. of Computer Science \\ The University of Vermont \\ Burlington, Vermont 05405 \\ 802-656-1918 \\ Maggie.Eppstein@uvm.edu
}

\begin{abstract}
Pair approximations have often been used to predict equilibrium conditions in spatially-explicit epidemiological and ecological systems. In this work, we investigate whether this method can be used to approximate takeover dynamics in spatially structured evolutionary algorithms. Our results show that the pair approximation, as originally formulated, is insufficient for approximating pre-equibilibrium dynamics, since it does not properly account for the interaction between the size and shape of the local neighborhood and the population size. After parameterizing the pair approximation to account for these influences, we demonstrate that the resulting system of differential equations can serve as a general and rapid approximator for takeover dynamics on a variety of spatially-explicit regular interaction topologies with varying population sizes. Strengths, limitations, and potential applications of the pair approximation to evolutionary computation are discussed.
\end{abstract}

\section{Categories and Subject Descriptors}

I.2.8 [Artificial Intelligence]: Problem Solving, Control Methods, and Search-Heuristic Methods

\section{General Terms}

Algorithms, Design, Experimentation, Performance

\section{Keywords}

Interaction topologies, pair approximations, saturation dynamics, spatial structure, takeover time analysis

\section{INTRODUCTION}

Most natural populations exhibit some form of spatial structure, and the important influence of the spatial scale of inter-individual interactions has thus become increasingly appreciated in many recent modeling efforts related to evolutionary biology, ecology, and epidemiology (e.g., [2, 4, 7, 8, $9,10,11,12,13,14,23])$. Spatially-localized interaction networks have also received an increasing amount of attention for use as population structures in evolutionary algorithms (e.g., $[1,5,15,17,19])$, because spatially localized interactions can help to maintain diversity by mitigating selection

Copyright is held by the author/owner(s).

GECCO'08, July 12-16, 2008, Atlanta, Georgia, USA.

ACM 978-1-60558-131-6/08/07. pressure. One commonly employed method for quantifying selective pressure in evolutionary algorithms is through the analysis of the dynamics with which a single favorable mutation spreads throughout the population (a.k.a. "takeover time analysis")[6]. While models of takeover dynamics have been developed for various types of regular (i.e., constant degree) local interaction neighborhoods embedded in Cartesion $2 \mathrm{D}$ space $[5,17,18]$, all of these models are specific to the particular interaction topologies for which they were designed.

Pair approximations (PAs) were originally derived as a statistical mechanics formulation of spatially structured biological populations, in order to determine conditions necessary for the evolution of altruism [11]. This technique has gained popularity in theoretical biology, ecology, and epidemiology $[2,7,8,9,13,16,20,21,22,23]$ due to its purported generality and the rapidity with which the resulting analytical expressions can be solved. In most cases, the PA has been used to predict equilibrium frequencies, in accordance with their original intent, although there have been some attempts to use them for estimating pre-equilibrium dynamics (e.g., $[8,16])$, with varying degrees of success.

Our intent is to investigate whether the PA can be used as a rapid and general method for approximating takeover dynamics of evolutionary algorithms structured on regular topologies with local interaction neighborhoods, based on a few readily computable metrics of the interaction network. Previously, we presented a PA of takeover dynamics [15], but this study was limited to a single population size. We have since determined that the PA, as originally formulated [11] and used by many others since, over-predicts the rate of spread of information, since it does not properly account for the interaction between the size and shape of the local neighborhood and the population size. After parameterizing the PA to account for these influences, we demonstrate that the resulting PA is an efficient approximator of takeover dynamics on a variety of spatially-explicit regular interaction topologies. We discuss the strengths, limitations, and potential improvements to the PA, and suggest how this approach may be useful to practitioners of evolutionary computation.

\section{METHODS}

A population structure is a graph $G$ of potential interactions between $\mu$ individuals located on vertices of the graph. If the population structure is regular, every vertex has the same degree $k$. In this study, we investigate takeover dynamics on ten distinct types of regular population structures, 
each based on 2D toroidal lattices, but with different local interaction neighborhoods (Table 1 ).

The clustering metric $(\phi)$ of a graph $G$ can be computed as the ratio of closed triangles to total triplets [8], as follows:

$$
\phi=\frac{\# \text { triangles }}{\# \text { triplets }}=\frac{\operatorname{trace}\left(A^{3}\right)}{\left\|A^{2}\right\|-\operatorname{trace}\left(A^{2}\right)}
$$

where the superscripts denote matrix exponentiation, $\left\|A^{2}\right\|$ denotes the sum of all of the elements in the square of the adjacency matrix $A$, and trace denotes the sum of the elements on the main diagonal. The radius of an interaction neighborhood $N$ captures the level of dispersion present in that neighborhood [19]. For an interaction neighborhood $N$ of size $k$, centered on a vertex located at $(x, y)$ in Cartesian space, this metric is formally defined as,

$$
\operatorname{radius}_{N}=\sqrt{\frac{1}{k}\left(\sum_{i=1}^{k}\left(x_{i}-\bar{x}\right)^{2}+\sum_{i=1}^{k}\left(y_{i}-\bar{y}\right)^{2}\right)}
$$

where

$$
\bar{x}=\frac{1}{k} \sum_{i=1}^{k} x_{i}, \quad \bar{y}=\frac{1}{k} \sum_{i=1}^{k} y_{i}
$$

The radius of the entire graph $\left(\right.$ radius $\left._{G}\right)$ can be calculated using equations (2) and (3) by assuming that the central vertex $\left(x=\frac{\sqrt{\mu}}{2}, y=\frac{\sqrt{\mu}}{2}\right)$ of graph $G$ is connected to every other vertex in the topology (i.e., $k=\mu-1$ ). The ratio of neighborhood to graph radius is denoted:

$$
\rho=\frac{\operatorname{radius}_{N}}{\operatorname{radius}_{G}}
$$

Table 2 presents the topological chacteristics of the ten types of neighborhood interaction structures used in this work, in order of increasing radius. These topologies represent the most commonly implemented regular, locally interacting, population structures used for spatially explicit evolutionary computation [5, 19], and modeling of ecological [4, 22] and evolutionary $[7,23]$ systems. We also implemented common variations of some of these, with larger interaction neighborhoods, such as extended Moore neighborhoods (M5, Table 1 and $M 7$, not shown) and extended Von Neumann neighborhoods (VN2, Table 1, and VN3, not shown).

In this study, we employ local uniform selection, where nodes are updated synchronously, as follows. For each node $i$, a node $j$ is selected at random with uniform probability from the mating neighborhood of node $i$, with neighborhood size $k$. Thus, if there are $x$ nodes containing the fittest value in the mating neighborhood of node $i$, then the probability of selecting one of them is simply $P_{\text {sel }}=\frac{x}{k}$. With uptake probability $p_{u p}$, the value of the selected node $j$ then replaces the value of node $i$ if node $j$ has higher fitness. High fitness individuals can also be allowed to mutate back to low fitness with some probability $g[18]$. Due to space constraints, the results reported are restricted to $p_{u p}=1$ and $g=0$, but this is not an inherent limitation of the pair approximation.

Each initial population comprises a single high fitness individual and $\mu-1$ low fitness individuals. If $N_{t}$ denotes the proportion of high fitness individuals at time $t$, then the takeover time of an individual experiment $T=\min \left\{t \mid N_{t}=\right.$ $1\}$ is defined as the minimum number of generations such that copies of the most fit individual fully saturate the entire population, starting with only one such individual in
Table 1: Naming conventions and schematic diagrams of the (a) rectangular and (b) triangular neighborhood structures considered in this study. (M7 and VN3 are not shown, but can be easily inferred). In (a), the links between vertices are implicit; each vertex in the interaction neighborhood (black circles) centered around a given vertex $(x)$ is connected to this center vertex. In (b), the links between individuals are shown explicitly (solid lines). (a)

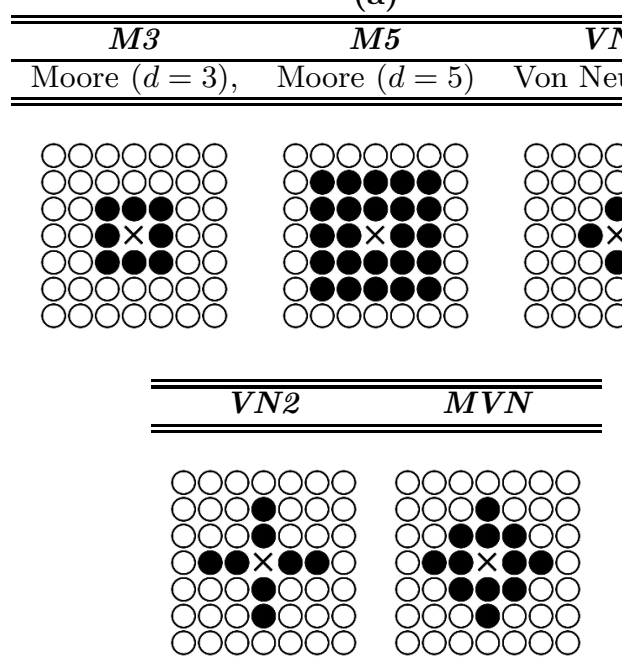

(b)

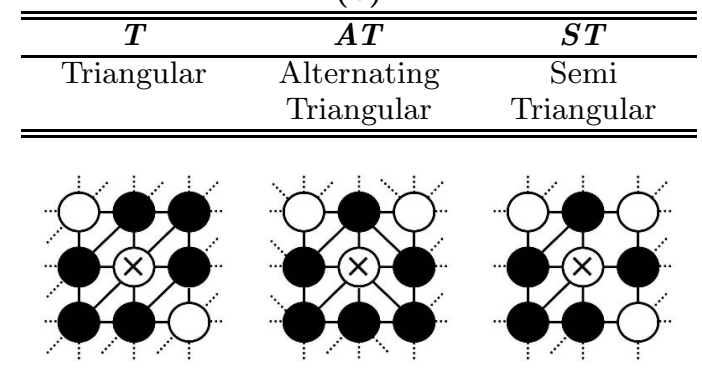

the initial population [17]. $E_{i}[T]$ is defined as the empirical estimate of the expected takeover time given that the initial best individual is located in vertex $i$, and the overall empirically estimated takeover time $E[T]$ is computed as the average of $E_{i}[T]$ over all vertices $i$ in the graph.

Instead of estimating the dynamics of the states of nodes in a contact network, pair approximations (PAs) estimate the dynamics of states of neighboring pairs of nodes. By capturing the correlations between pairs of vertices, some aspects of the structure of the interaction topology can be accounted for. Consider a population of size $\mu$ structured on an interaction topology wherein every node has $k$ neighbors. (It is important to note that PAs assume that the underlying contact network is regular, or at least possesses a well defined average degree $k$.) Following [8], let $[X]$ denote the number of nodes in state $X,[X Y]$ denote the number of pairs of connected nodes in state $X Y$, and $[X Y Z]$ denote the number of connected triplets of nodes in state $X Y Z$, such 
Table 2: Structural metrics of the spatial interaction topologies considered in this study, presented in order of increasing radius of the local neighborhoods.

\begin{tabular}{cccc}
\hline \hline Population Structure & $k$ & $\phi$ & radius $_{N}$ \\
\hline$V N 1$ & 4 & 0 & 0.89 \\
$S T$ & 5 & 0.3 & 1.06 \\
$A T$ & 6 & 0.4 & 1.11 \\
$T$ & 6 & 0.4 & 1.16 \\
M3 & 8 & 0.43 & 1.23 \\
MVN & 12 & 0.46 & 1.47 \\
VN2 & 8 & 0.21 & 1.49 \\
M5 & 24 & 0.52 & 2.00 \\
$V N 3$ & 12 & 0.27 & 2.08 \\
M7 & 48 & 0.54 & 2.83 \\
\hline
\end{tabular}

that $X Y$ pairs are always counted once in each direction (i.e., $[X Y]=[Y X])$ and $X X$ pairs are counted twice (i.e., $[X X]$ is always even). PAs work by tracking the changes in the numbers of all possible combinations of pairs $[X Y]$. Since the interaction topology is regular with constant degree $k$, the number of singles can always be recovered from the number of pairs, as follows [8]:

$$
[X]=\frac{1}{k} \sum_{W}[X W]
$$

However, the rates of change in the number of pairs depend upon the numbers of configurations larger than pairs, such as triplets. Thus, in order to estimate the dynamics in terms of the numbers of pairs, the numbers of configurations larger than pairs must be approximated to some degree of accuracy; this is referred to as "closing" the system $[8,11$, $22]$. We employ the closure method proposed in [8], which incorporates the clustering coefficient (Equation 1), accounting for the proportion of triplets in the interaction topology that form closed triangles [8], as follows:

$$
[X Y Z] \approx \frac{(k-1)}{k} \frac{[X Y][Y Z]}{[Y]}\left((1-\phi)+\frac{\phi \mu}{k} \frac{[X Z]}{[X][Z]}\right)
$$

Spatial structure beyond triplets, however, is not considered in equation (6).

We modify the 5-equation Susceptible-Infectious-Recovered (SIR) PA proposed by [8] into a 3-equation model of takeover dynamics (which is equivalent to an SIS model [15]), as follows:

$$
\begin{aligned}
\frac{d[S S]}{d t} & =c\left(-\frac{p_{u p}}{k}[S S I]+g[S I]+g^{2}[I I]\right) \\
\frac{d[S I]}{d t} & =0.5 c\left(\frac{p_{u p}}{k}([S S I]-[I S I]-[S I])+g([I I]-[S I])\right) \\
\frac{d[I I]}{d t} & =c\left(\frac{p_{u p}}{k}([I S I]+[S I])-g[I I]-g^{2}[I I]\right)
\end{aligned}
$$

where vertices can be in either state $I$ for high fitness (i.e., Infectious) or $S$ for low fitness (i.e., Susceptible), and the factor of 0.5 in the second equation accounts for the symmetry between $[S I]$ and $[I S]$. In the original derivation of the PA [11], designed for the prediction of population densities at equilibrium, the value of the coefficient $c$ was proposed to be the constant 2 , and this value has since been used in numerous other studies (e.g., $[8,16,21,22])$. In fact, it is trivial to show that equilibrium conditions are independent of the particular choice of the coefficient $c$, as long as the relative values of the coefficients of the equations for $[S S]:[S I]:[I I]$ are 2:1:2. However, if one is to use the PA to approximate pre-equilbrium dynamics, then the absolute value of $c$ also becomes important. As will be shown in Section 3, our results clearly demonstrate that the optimal choice of $c$ is a function of $\rho$ (Equation (4)), which depends on both the local neighborhood of interactions and the population size.

For each combination of the ten population structures (Table 2) and eight population sizes $(\mu \in\{576,1024,1600,2304$, $3136,4096,5184,6400\})$, takeover dynamics were observed by placing a single copy of the best individual in only one node and then observing the rate with which this advantageous allele spreads through the population. For each of the 80 distinct combinations of population structure and population size, 50 such simulations were performed and averaged, in order to mitigate the stochasticity inherent in the selection policy. Takeover dynamics were also approximated by solving the coupled differential equations of the PA (System of Equations (7)) via numerical integration, using a Runga-Kutta method with adaptive step size (Matlab's ode45 function). Since the PA is a continuous approximation, the expected takeover times of both the simulation data and the PA predictions were calculated as the first generation in which $N_{t}$ was within $1 \%$ of the maximum saturation value of 1. Generation error is defined as the maximum absolute generation difference, for the same degree of saturation $N_{t}$, between the PA and simulation curves, expressed as a percentage of the takeover time observed through simulation $\left(E[T]_{\text {Sim }}\right)$.

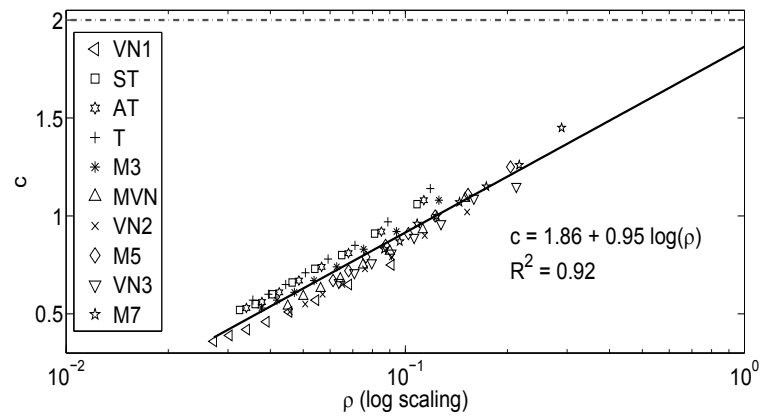

Figure 1: The values of $c$ that minimized generation error as a function of $\rho$, along with the best logarithmic fit. The dash-dot horizontal line, representing $c=2$, is provided as reference.

\section{RESULTS}

The optimal values for $c$ which minimize generation errors increase logarithmically with $\rho\left(R^{2}=0.92\right.$, Figure 1), according to the best-fit equation:

$$
c \approx 1.86+0.95 \log (\rho)
$$

This relationship is consistent with the findings of [19], who reported that the rate of saturation increased logarithmically with the ratio of local neighborhood radius to grid radius. The value of $c=2$, used in previous studies, is shown for reference (Figure 1, horizontal dash-dot line); in 

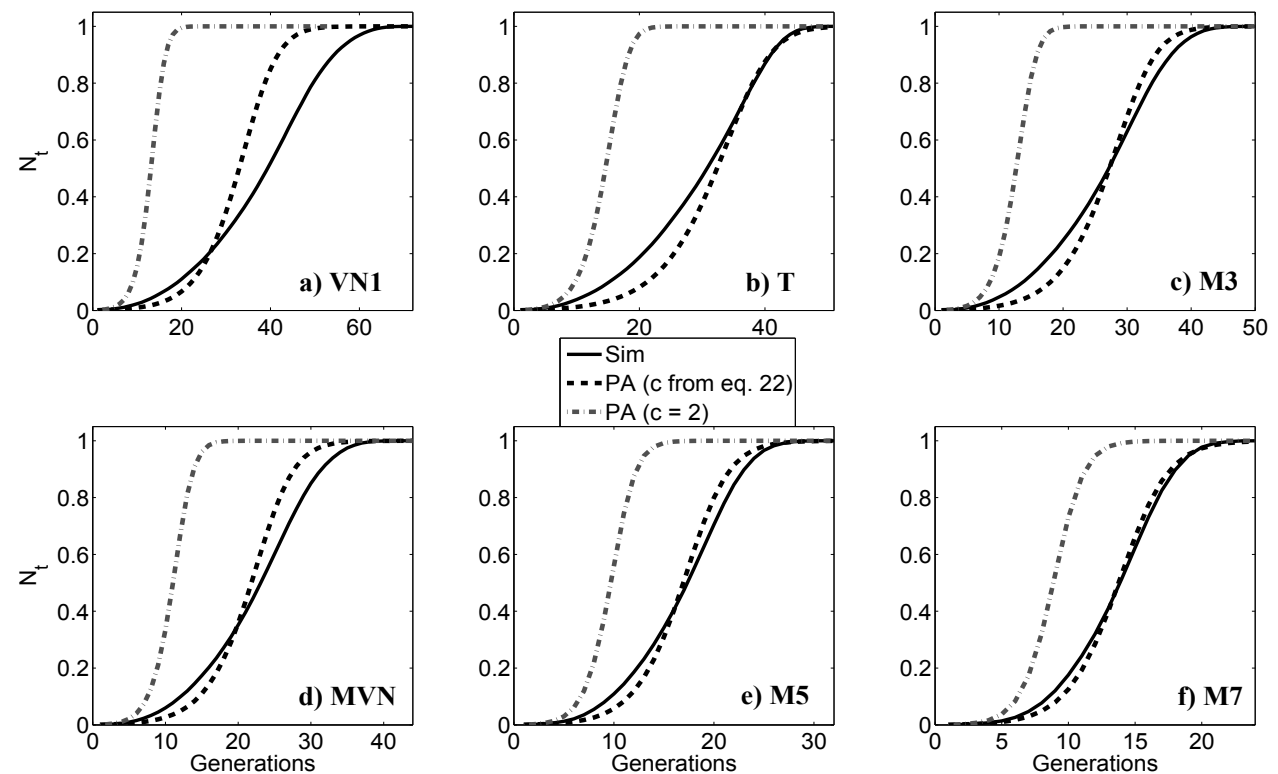

Figure 2: Takeover dynamics as predicted by the PA using equation (8) (dashed black lines) and as observed through direct simulation (solid black lines) on six regular population structures using $\mu=1024$. For reference, we also show the dynamics predicted by the PA using $c=2$ (dash-dot lines). The legend and vertical axis applies to all panels. Note the change in scale among the horizontal axes of each panel.

all cases, the optimal coefficient values for $c$ were well below 2. Equation (8) was used in all subsequent experiments, to assess the accuracy of the PA (System of Equations (7)) with the coefficient $c$ parameterized by $\rho$.

Takeover time was found to decrease as the radius of the local neighborhood increased, in concurrence with the results of [19]. Figure 2 shows takeover dynamics predicted by the PA (dashed black lines) and observed by direct simulation (solid black lines) on six representative regular population structures with $\mu=1024$. When plotting simulation results here and elsewhere in this paper, we depict the proportion of nodes $\left(N_{t}\right)$ containing maximum fitness at generation $t$, averaged over all 50 independent simulations on that combination of graph type and population size.

Takeover curves for the PA are sigmoidal, exhibiting exponential growth $\left(R^{2}>0.95\right.$ for an exponential fit below the inflection point, for all PA curves) followed by saturation. In contrast, takeover curves on 2D lattice topologies with local interaction neighborhoods are known to be polynomial [5] below the inflection point. Polynomial exponent varied from 1.9 to $2.8\left(R^{2}>0.96\right.$, for all topologies and population sizes considered herein), where the exponent increased linearly with increasing radius $_{N}\left(R^{2}>0.96\right.$ for all population sizes). Thus, the PA is not an actual mechanistic model of the governing dynamics of this system, but is more appropriately characterized as an approximation, and all PA curves were statistically different from the corresponding simulation curves $\left(p<0.001, \chi^{2}\right)$. However, in most cases the resulting curves are in reasonably good agreement with the data observed through simulation (Figure 2c-f). In contrast, the curves resulting from the PA using $c=2$, shown in Figure 2 as dash-dot lines, always dramatically overestimate the rate of spread of the advantageous allele.

If the optimal $c$ were used, the simulation and PA curves would intersect at the inflection point. However, the use of equation (8) to estimate $c$ introduces a small amount of error, sometimes causing the curves to intersect a little too early (as for VN1, Figure 2a), or a little too late (as for T, Figure $2 \mathrm{~b}$ ), thus resulting in an over- or under-estimate of the rate of takeover, respectively. Other errors are due to simplifications in the PA itself, causing the PA to be least accurate on the VN1 topology, and most accurate for populations with large neighborhood radii and small population sizes. These results are quantified below and discussed in Section 4.

Generation error (Figure 3) was found to decrease approximately exponentially as a function of $\rho$ for each distinct population structure $\left(0.83<R^{2}<0.99\right.$, with an average of $\left.R^{2}=0.91\right)$. The exponential decrease of generation error as $\rho$ increases has the ironic implication that the PA, even after the formulation has been adjusted to compensate for $\rho$, becomes more accurate for estimating saturation dynamics as the system approaches the well-mixed case, where population growth becomes sigmoidal. This finding also has the important implication that for any given local interaction neighborhood, the accuracy of the PA depends heavily upon population size $(\mu)$, with smaller population sizes yielding more accurate results. For example, as population size was increased from $\mu=1024$ to $\mu=6400$ on the $M 3$ topology, generation error increasing from $10 \%$ to $18 \%$, be- 
cause as takeover times increase the exponential growth of the PA curve has more time to diverge from the polynomial growth of the simulation curve.

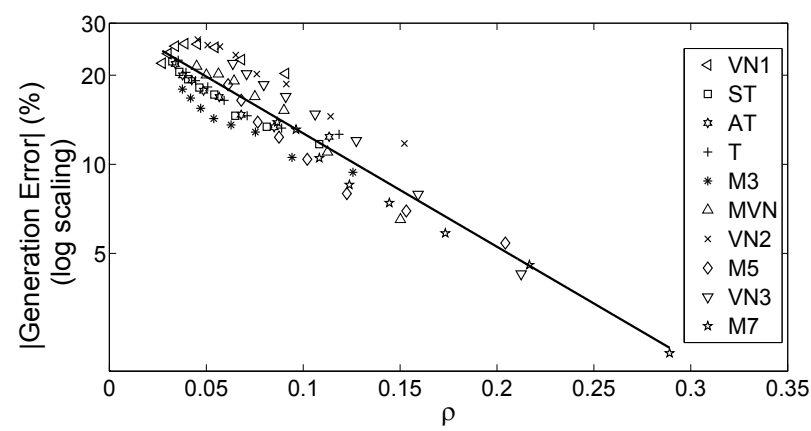

Figure 3: Generation error as a function of $(\rho)$, for all population sizes and population structures. The solid line depicts the best exponential fit to the data for all population structures, and is provided as a visual aid only. Note the logarithmic scale on the vertical axis.

\section{DISCUSSION}

The aim of this study was to investigate whether a pair approximation (PA) could be used to easily and accurately estimate takeover dynamics for evolutionary algorithms that are spatially-structured on regular graphs. The original presentation of the PA [11], which was designed to approximate equilibrium conditions in spatially structured biological populations in order to determine conditions for the evolution of altruism [11], incorporated a constant coefficient of $c=2$. This value has since been used in numerous other studies (see Section 1). While the value of this coefficient does not affect equilibrium frequencies, we show that it does dramatically impact predictions of pre-equilibrium dynamics. Our results demonstrate that, in all ten population structures tested, a value of $c=2$ caused the PA to dramatically overestimate the rate of spread of advantages alleles, consistent with the results presented by [16] on three population structures with fixed population sizes. Furthermore, we show that, if one is trying to predict pre-equilibrium dynamics, the value of the PA coefficient $c$ that minimizes generational errors depends on the interaction between (a) the structure of the local interaction neighborhood and (b) the population size. We combine these two influences into a single parameter $\rho$ (defined as the ratio of the radius of the local interaction neighborhood to the radius of the entire population) and show that the optimal value for the coefficient $c$ can be estimated as a logarithmic function of $\rho$. Parameterizing the coefficient $c$ by $\rho$ effectively shifts the saturation curve predicted by the PA so that it intersects with the simulation data at (or near) the inflection point, thereby minimizing generational errors in the PA. In general, two primary sources of error remain: (a) residual errors introduced by using the regression curve to estimate $c$, and (b) errors introduced by the simplifications in the PA itself, as discussed below.

The PA was assessed using the values of $c$ predicted by the empirically derived logarithmic fit relating $c$ to $\rho$. Although this fit was quite good $\left(R^{2}>0.92\right)$, individual values of $c$ selected according to this formula did contain some small residual error, which caused some predicted curves to intersect the simulation curve a little before the inflection points, thus causing a slight net over-prediction in the rate of spread, or a little after the inflection points, causing a slight net under-prediction in the rate of spread.

The PA uses differential equations to model the dynamics of states of neighboring pairs of vertices. Higher order interactions are not explicitly modeled, but must be approximated using some method of closure. We employed the closure method of [8], which incorporates the proportion of closed to total triplets that exist in the local interaction neighborhood (a.k.a. the clustering coefficient), thus closing the system at the level of triplets. Consequently, the PA yielded less accurate predictions of dynamics on populations with Von Neumann neighborhoods, which have no closed triangles (and thus no clustering) but do have a preponderance of closed quadruplets. By ignoring quadruplet correlations and assuming no correlation between distant ends of triplets, the PA treats the $V N 1$ population structure as if it were a regular random graph with degree $k$. This explains the more rapid saturation predicted by the PA than that observed through direct simulation. Thus, accuracy could be improved by explicitly accounting for these correlations using higher order closure methods [20, 22] or higher order approximations [16]. While these higher-order methods have yet to be explored on different population sizes, our results suggest that the interaction between neighborhood size and shape and population size will still need to be accounted for. Such higher-order improvements could be used in combination with a paramterized coefficient $c$, as suggested in this manuscript, although the best fit curve for this would need to be recomputed for a given approximation method.

The accuracy of the PA decreases exponentially with increasing $\rho$ (i.e., with increasing neighborhood interaction radii and/or decreasing population size), in part because larger neighborhood radii have higher polynomial exponents, and in part because, as population sizes are decreased, individual neighborhoods cover a larger proportion of the population and thus the system more closely approaches random mixing. Furthermore, as population size increases, the exponential growth of the PA curve has more time to diverge from the polynomial growth of the simulation curve, thereby increasing discrepancies between the two curves. It is ironic that PAs, which were designed to model local interactions, actually work better when interactions are far-ranging. It is also usually assumed that continuous models of discrete systems work better as population sizes approach infinity, but in this case the PA works better for small populations, a counter-intuitive finding.

The local uniform selection mechanism employed herein is clearly a simplification of the selection operators that are commonly used in evolutionary algorithms. However, PAs can be modified to employ much more complicated selection policies. For example, PAs have been developed using frequency dependent selection [23] and game-theoretic payoff matrices $[7,13]$. The PA could be similarly adjusted to include a more sophisticated reversion mechanism as well (e.g., frequency dependence), and recent work [3] has demonstrated that the PA can even be adjusted to deal with processes that operate on differing spatial scales.

In summary, the results of this study demonstrate that our parameterized formulation of the PA is a fast and reasonably accurate way to estimate both equilibrium and pre- 
equilibrium takeover characteristics of synchronously updated populations embedded on a variety of regularly structured graphs. A key result of this study is that the coefficient $c$ in the PA (which is commonly assumed to be $c=2$ ) should be parameterized by $\rho$, if one is interested in approximating pre-equilibrium conditions. In evolutionary computation, population structures are user-defined, frequently regular, and their topological properties are readily computable. We conclude that PAs can be a useful tool for rapidly estimating takeover dynamics in evolutionary algorithms on synchronously updated regular graphs, as long as care is taken to assess the topological characteristics of the graph in advance and the PA is appropriately formulated. Future work will seek to demonstrate if such insights may prove useful for guiding choices of local neighborhood structures in evolving populations, as a potential means of statically or dynamically optimizing selection pressure and convergence.

\section{ACKNOWLEDGEMENTS}

We thank Mario Giacobini for helpful comments and suggestions which contributed to our improving this work. Presentation of this work was funded in part by Vermont EPSCoR (EPS 0701410).

\section{REFERENCES}

[1] K. M. Bryden, D. Ashlock, S. Corns, and S. Wilson. Graph based evolutionary algorithms. IEEE Transactions on Evolutionary Computation, 10(5):550-567, 2005.

[2] K. T. D. Eames and M. J. Keeling. Modeling dynamic and network heterogeneities in the spread of sexually transmitted diseases. Proceedings of the National Academy of the Sciences, 99(20):13330-13335, 2002.

[3] S. P. Ellner. Pair approximations for lattice models with multiple interaction scales. Journal of Theoretical Biology, 210(4):435-447, 2001.

[4] M. J. Eppstein and J. Molofsky. Invasiveness in plant communities with feedbacks. Ecology Letters, 10:253-263, 2007.

[5] M. Giacobini, M. Tomassini, A. Tettamanzi, and E. Alba. Selection intensity in cellular evolutionary algorithms for regular lattices. IEEE Transactions on Evolutionary Computation, 9(5):489-505, 2005.

[6] D. Goldberg and K. Deb. A comparative analysis of selection schemes used in genetic algorithms. In G. Rawlins, editor, Foundations of Genetic Algorithms, pages 69-93. Morgan Kaufmann, San Francisco, 1991.

[7] C. Hauert and M. Doebeli. Spatial structure often inhibits the evolution of cooperation in the snowdrift game. Nature, 428:643-646, 2004.

[8] M. J. Keeling. The effects of local spatial structure on epidemiological invasions. Proceedings of the Royal Society of London B, 266:859-867, 1999.

[9] M. J. Keeling and K. T. D. Eames. Networks and epidemic models. Journal of the Royal Society Interface, 2:295-307, 2005.

[10] B. Kerr, M. A. Riley, M. W. Feldman, and B. J. M. Bohannan. Local dispersal promotes biodiversity in a real life game of rock-paper-scissors. Nature, 418:171-174, 2002.
[11] H. Matsuda, N. Ogita, A. Sasaki, and K. Soto. Statistical mechanics of population: the lattice lotka-volterra model. Progress of Theoretical Physics, 88:1035-1049, 1992.

[12] M. E. J. Newman. Spread of epidemic disease on networks. Physical Review E, 66:016128, 2002.

[13] H. Ohtsuki, C. Hauert, E. Lieberman, and M. A. Nowak. A simple rule for the evolution of cooperation on graphs and social networks. Nature, 441:502-505, 2006.

[14] J. L. Payne and M. J. Eppstein. Sensitivity of self-organized speciation to long-distance dispersal. In Proceedings of the IEEE Symposium on Artificial Life, pages 1-7, 2007.

[15] J. L. Payne and M. J. Eppstein. Using pair approximations to predict takeover dynamics in spatially structured populations. In D. Thierens, editor, Proceedings of the Genetic and Evolutionary Computation Conference, GECCO-200\%. ACM Press, New York, 2007. Late-Breaking Papers.

[16] T. Petermann and P. De Los Rios. Cluster approximations for epidemic processes: a systematic description of correlations beyond the pair level. Journal of Theoretical Biology, 229:1-11, 2004.

[17] G. Rudolph. On takeover times in spatially structured populations: array and ring. In K. Lai, O. Katai, and a. B. L. M. Gen, editors, Proceedings of the Second Asia-Pacific Conference on Genetic Algorithms and Applications, APGA-2000, pages 144-151. Global Link Publishing Company, Hong Kong, 2000.

[18] G. Rudolph. Takeover times of noisy non-generational selection rules that undo extinction. In V. Kurkova, editor, Proceedings of the Fifth International Conference on Artificial Neural Networks and Genetic Algorithms, pages 268-271. Springer-Verlag, Heidelberg, 2001.

[19] J. Sarma and K. De Jong. An analysis of the effect of neighborhood size and shape on local selection algorithms. In H. Voigt, W. Ebeling, I. Rechenberg, and H. Schwefel, editors, Parallel Problem Solving from Nature, pages 236-244. Springer-Verlag, Heidelberg, 1996.

[20] K. Satō and Y. Iwasa. Pair approximations for lattice-based ecological models. In U. Dieckmann, R. Law, and J. A. J. Metz, editors, The Geometry of Ecological Interactions: Simplifying Spatial Complexity, pages 341-358. Cambridge University Press, Cambridge, 2000.

[21] K. Satō, H. Matsuda, and A. Sasaki. Pathogen invasion and host extinction in lattice structured populations. Journal of Mathematical Biology, 32:251-268, 1994.

[22] M. Van Baalen. Pair approximations for different spatial geometries. In U. Dieckmann, R. Law, and J. A. J. Metz, editors, The Geometry of Ecological Interactions: Simplifying Spatial Complexity, pages 359-387. Cambridge University Press, Cambridge, 2000.

[23] M. Van Baalen and D. A. Rand. The unit of selection in viscous populations and the evolution of altruism. Journal of Theoretical Biology, 193:631-648, 1998. 